\title{
A
}

Acta HealthMedica

Acta HealthMedica (ISSN: 2414-6528)

http://www.ActaHealthMedica.com

Volume: 2, Issue: 1, January-March 2017, Pages: 112, DOI: http://dx.doi.org/10.19082/ah112

\section{DECISION SUPPORT ALGORITHMS FOR CLINICAL EVALUATION OF EEG; EXPERIENCE OF ADHD CHILDREN}

\author{
Marjan Ghazisaeedi ${ }^{1}$, Leila Shahmoradi ${ }^{2}$, Sharareh R. Niakan Kalhori ${ }^{3}$, Azadeh Bashiri*4
}

1: Assistant Professor, Department of Health Information Management, School of Allied Medical Sciences, Tehran University of Medical Sciences, Tehran, Iran

2: Assistant Professor, Department of Health Information Management, School of Allied Medical Sciences, Tehran University of Medical Sciences, Tehran, Iran

3: Assistant Professor, Department of Health Information Management, School of Allied Medical Sciences, Tehran University of Medical Sciences, Tehran, Iran

4: Ph.D Candidate, Department of Health Information Management, School of Allied Medical Sciences, Tehran University of Medical Sciences, Tehran, Iran

Corresponding author:

Azadeh Bashiri. Health Information Management Department, School of Allied Medical Sciences, Quds Street, Post Code: 1594733711, Tehran, Iran. Tel: +989175014231, E-mail: a-bashiri@razi.tums.ac.ir

\section{TYPE OF ARTICLE: CONFERENCE ABSTRACT}

\begin{abstract}
Introduction: Attention deficit hyperactivity disorder (ADHD), as one of the most common neuro-behavioral disorders, is caused as a result of brain dysfunction and, depending on the age and frequency, can heavily affect the electroencephalography (EEG). Subjective and overlapping behavioral symptoms of ADHD with other psychiatric disorders are long-standing issues that have an effect on the diagnostic process. Thus, it is natural that the EEG as an objective measurement is used to evaluate the clinical status of these patients. The aim of this study is to highlight the using of machine learning techniques to analyze the EEG in clinical evaluations of ADHD children.

Methods: This review article has been conducted by searching articles in scientific databases and e-Journals and by using keywords that include EEG, machine learning techniques, decision support system (DSS), and ADHD.

Results: EEG signals are produced as a result of neural activity and are based on the frequency divided in fourbands (delta, theta, alpha, and beta). Studies have shown the abnormalities of EEG signals such as lower frequency of alpha, the higher frequency of delta and theta and the epileptiform patterns in ADHD children. In order to understand the nature of EEG in the evaluation of these individuals, linear and nonlinear classifiers, including support vector machines, artificial neural networks, and bayesian networks, have been used. These classification methods have the ability to handle multidimensional features and nonlinear relationships in EEG data. They improve the accuracy of diagnosis process and differentiate between the ADHD and control groups as well as the subtypes of ADHD.
\end{abstract}

Conclusion: Today, there are a lot of concerns about exaggerating diagnoses and unnecessary medication therapy of individuals with ADHD. The aim of EEG studies in ADHD patients is to explore data related to various aspects of brain functions. The high sensitivity and accuracy of these data are useful in evaluation and differential diagnosis of this disorder. Thus, designing decision support systems based on machine learning techniques for analyzing EEG has the high applicability to offer more accurate diagnosis and improve the rehabilitation of ADHD children.

KEYWORDS: Machine learning, Electroencephalography, Attention deficit hyperactivity disorder, Decision support system

\section{Abstracts of First National Congress of Medical Informatics, Mashhad, Iran, February 2017}

(C) 2017 The Authors. This is an open access article under the terms of the Creative Commons Attribution-NonCommercialNoDerivs License, which permits use and distribution in any medium, provided the original work is properly cited, the use is non-commercial and no modifications or adaptations are made. 